\title{
Article
}

\section{Repdigits as Product of Terms of $k$-Bonacci Sequences}

\author{
Petr Coufal ${ }^{1}$ a and Pavel Trojovský ${ }^{2, *}$ (1) \\ 1 Department of Applied Cybernetics, Faculty of Science, University of Hradec Králové, \\ 50003 Hradec Králové, Czech Republic; petr.coufal@uhk.cz \\ 2 Department of Mathematics, Faculty of Science, University of Hradec Králové, \\ 50003 Hradec Králové, Czech Republic \\ * Correspondence: pavel.trojovsky@uhk.cz; Tel.: +42-049-333-2860
}

check for

updates

Citation: Coufal, P.; Trojovský, P. Repdigits as Product of Terms of $k$-Bonacci Sequences. Mathematics 2021, 9, 682. https://doi.org/ $10.3390 /$ math 9060682

Academic Editors: Yang-Hui He and Rekha Srivastava

Received: 25 February 2021

Accepted: 21 March 2021

Published: 22 March 2021

Publisher's Note: MDPI stays neutral with regard to jurisdictional claims in published maps and institutional affiliations.

Copyright: (c) 2021 by the authors. Licensee MDPI, Basel, Switzerland. This article is an open access article distributed under the terms and conditions of the Creative Commons Attribution (CC BY) license (https:/ / creativecommons.org/licenses/by/ $4.0 /)$.

\begin{abstract}
For any integer $k \geq 2$, the sequence of the $k$-generalized Fibonacci numbers (or $k$-bonacci numbers) is defined by the $k$ initial values $F_{-(k-2)}^{(k)}=\cdots=F_{0}^{(k)}=0$ and $F_{1}^{(k)}=1$ and such that each term afterwards is the sum of the $k$ preceding ones. In this paper, we search for repdigits (i.e., a number whose decimal expansion is of the form $a a \ldots a$, with $a \in[1,9])$ in the sequence $\left(F_{n}^{(k)} F_{n}^{(k+m)}\right)_{n}$, for $m \in[1,9]$. This result generalizes a recent work of Bednařík and Trojovská (the case in which $(k, m)=(2,1))$. Our main tools are the transcendental method (for Diophantine equations) together with the theory of continued fractions (reduction method).
\end{abstract}

Keywords: k-generalized Fibonacci numbers; linear forms in logarithms; reduction method

MSC: 11A63; 11B37; 11B39; 11J86

\section{Introduction}

We start by recalling that the Fibonacci sequence $\left(F_{n}\right)_{n}$ is defined by the recurrence

$$
F_{n+1}=F_{n}+F_{n-1}
$$

with initial values $F_{0}=0$ and $F_{1}=1$ (see, e.g., [1-3]). This sequence admits many generalizations and one of the most known is its higher order version. The Fibonacci sequence is a binary (or second order) recurrence and then, for any integer $k \geq 2$, the sequence of the $k$-generalized Fibonacci numbers (or $k$-bonacci numbers) is defined by the $k$ th order recurrence

$$
F_{n}^{(k)}=F_{n-1}^{(k)}+\cdots+F_{n-k}^{(k)}
$$

with initial values $F_{-(k-2)}^{(k)}=\cdots=F_{0}^{(k)}=0$ and $F_{1}^{(k)}=1$. Clearly, for $k=2$, we have the Fibonacci numbers and for $k=3$, we have Tribonacci numbers (which is one of the most well-studied generalizations of Fibonacci numbers).

On the other hand, a repdigit (short for "repeated digit") is a number of the form

$$
a\left(\frac{10^{\ell}-1}{9}\right)
$$

where $\ell \geq 1$ and $a \in[1,9]$ (here, as usual, for integers $x<y$, we denote $[x, y]=$ $\{x, x+1, \ldots, y\}$ ), that is, a number with only one distinct digit (in this case $a$ ) in its decimal expansion.

We point out that many authors have been interested in solving Diophantine equations involving repdigits (their sums, products, concatenations, etc.) and some special forms of linear recurrences (like their product, sums, etc.). For some works in this direction, we refer the reader to [4-33] and the references therein. 
Luca [34], in 2000, and Marques [35], in 2012, proved that the largest repdigits in the Fibonacci and Tribonacci sequence are $F_{10}=55$ and $T_{8}=44$, respectively. Recently, Bednařík and Trojovská [36] and Trojovský [37] found all repdigits of the form $F_{n} T_{n}$ and $F_{n}+T_{n}$, respectively.

The aim of this paper is to continue this program and generalize the main result of [36]. More precisely, we search for repdigits which are the product of the $n$th $k$-bonacci number by the $n$th $(k+m)$-bonacci number, for $m \in[1,9]$. Our main result is the following:

Theorem 1. The Diophantine equation

$$
F_{n}^{(k)} F_{n}^{(k+m)}=a\left(\frac{10^{\ell}-1}{9}\right)
$$

does not have a solution in positive integers $n, a, k, \ell$, with $k \geq 2, \ell>1$ and $a, m \in[1,9]$.

The main tools in the proof is the transcendental method (lower bounds for linear logarithm of real algebraic numbers) together with the theory of continued fractions (reduction method). It is important to stress that the method can be implemented for any given range of values for $m$. However, we chose $m \in[1,9]$ in order to avoid too much time of computation (by using Mathematica software).

\section{Auxiliary Results}

The results of this section can be found in the classical literature about this kind of Diophantine equation (see, for example, [20] and the references therein). For this reason, we shall present here these tools as succinctly as possible.

The first useful result is due to Dresden and Du [38] (Theorem 1) who proved that

$$
F_{n}^{(k)}=g(\alpha, k) \alpha^{n-1}+E_{n, k}
$$

with $\left|E_{n, k}\right|<1 / 2$, where $\alpha$ is the dominant root of the polynomial $\psi_{k}(x):=x^{k}-\sum_{j=0}^{k-1} x^{j}$. Moreover, we have the notation $g(x, y):=(x-1) /(2+(y+1)(x-2))$. Furthermore, Bravo and Luca [39] showed that

$$
\alpha^{n-2} \leq F_{n}^{(k)} \leq \alpha^{n-1} .
$$

Another very useful ingredient is the following result à la Baker:

Lemma 1. Let $\gamma_{1}, \ldots, \gamma_{t} \in \mathbb{R}$ be algebraic numbers and let $b_{1}, \ldots, b_{t}$ be nonzero integer numbers. Let $D$ be the degree of the number field extension $\mathbb{Q}\left(\gamma_{1}, \ldots, \gamma_{t}\right) / \mathbb{Q}$ and let $A_{j} \in$ be any constant such that

$$
A_{j} \geq \max \left\{D h\left(\gamma_{j}\right),\left|\log \gamma_{j}\right|, 0.16\right\}, \text { for } j \in[1, t] .
$$

In addition, choose a constant $B$ for which

$$
\begin{aligned}
& B \geq \max \left\{\left|b_{1}\right|, \ldots,\left|b_{t}\right|\right\} . \\
& \text { If } \gamma_{1}^{b_{1}} \cdots \gamma_{t}^{b_{t}} \neq 1 \text {, then } \\
& \left|\gamma_{1}^{b_{1}} \cdots \gamma_{t}^{b_{t}}-1\right| \geq \exp \left(-1.4 \cdot 30^{t+3} \cdot t^{4.5} \cdot D^{2}(1+\log D)(1+\log B) A_{1} \cdots A_{t}\right) .
\end{aligned}
$$

This result is a version of a Matveev theorem [40] due to Bugeaud et al. (see its proof in [41]). In the previous statement, $\mathrm{h}(\gamma)$ denotes the logarithmic height of an $\ell$-degree algebraic number $\gamma$. This function satisfies the following properties (the proof of these facts can be found in [42]): 
Lemma 2. We have

$$
\begin{array}{ll}
\text { i. } & \mathrm{h}(x y) \leq \mathrm{h}(x)+\mathrm{h}(y) ; \\
\text { ii. } & \mathrm{h}(x+y) \leq \mathrm{h}(x)+\mathrm{h}(y)+\log 2 ; \\
\text { iii. } & \mathrm{h}\left(\alpha^{r}\right)=|r| \cdot \mathrm{h}(\alpha), \text { for all } r \in \mathbb{Z} .
\end{array}
$$

Finally, the last tool was proved by Dujella and Pethő [43] (Lemma 5(a)):

Lemma 3. Let $M$ be a positive integer and let $p / q$ be a convergent of the continued fraction expansion of the irrational number $\gamma$ such that $q>6 M$. Let $(A, B) \in \mathbb{R}_{>0} \times \mathbb{R}_{>1}$ and define $\epsilon:=\|\mu q\|-M\|\gamma q\|$, where $\mu$ is a given real number. If $\epsilon>0$, then there is no solution to the Diophantine inequality

$$
0<m \gamma-n+\mu<A \cdot B^{-k}
$$

in positive integers $m, n$ and $k$ satisfying

$$
m \leq M \text { and } k \geq \frac{\log (A q / \epsilon)}{\log B} .
$$

In the previous statement, we used the notation $\|x\|=\min \{|x-n|: n \in \mathbb{Z}\}$.

Now, we are ready to deal with the proof of the theorem.

\section{The Proof of Theorem 1}

\subsection{Upper Bounds for $n$ and $\ell$ in Terms of $k$}

Let $\alpha$ and $\beta$ be the dominant roots of the sequences $\left(F^{(k)}\right)_{n}$ and $\left(F^{(k+m)}\right)_{n}$, respectively. Moreover, set $g:=g(\alpha, k), h:=g(\beta, k+m), e_{1}:=E_{n, k}$ and $e_{2}:=E_{n, k+m}$. Thus, by using (4) in Equation (3), we obtain

$$
\left(g \alpha^{n-1}+e_{1}\right)\left(h \beta^{n-1}+e_{2}\right)=a\left(\frac{10^{\ell}-1}{9}\right) .
$$

After some computations, we get

$$
\left|g h(\alpha \beta)^{n-1}-a \frac{10^{\ell}}{9}\right|<3 \beta^{n-1},
$$

where we used that $\max \left\{\left|e_{1}\right|,\left|e_{2}\right|\right\}<1 / 2$ and $\alpha<\beta$. Now, we divide the inequality by $g h(\alpha \beta)^{n-1}$, to derive

$$
\left|1-a(9 g h)^{-1}(\alpha \beta)^{n-1} 10^{\ell}\right|<\frac{96}{\alpha^{n}}
$$

where we applied the inequality $\min \{|g|,|h|\}>1 / 4$ (see [44]). Let us define

$$
\Lambda=\ell \log 10-(n-1) \log (\alpha \beta)+\log \theta_{a, m},
$$

where $\theta_{a, m}:=a(9 g h)^{-1}$ (for $\left.a, m \in[1,9]\right)$. Then, Equation (8) can be rewritten as

$$
\left|e^{\Lambda}-1\right|<\frac{96}{\alpha^{n}}
$$

First, we claim that $\Lambda \neq 0$ (for $\ell>1$ ). On the contrary, we would have $a 10^{\ell} / 9=$ $g h(\alpha \beta)^{n-1}$. Now, we can apply to the previous equality, a nontrivial automorphism $\sigma: \mathbb{K} \rightarrow \mathbb{K}\left(\alpha \mapsto \alpha_{2}\right.$ and $\left.\beta \mapsto \beta_{2}\right)$ of the Galois group of $\mathbb{K}:=\mathbb{Q}(\alpha, \beta)$ to obtain (after applying absolute values): 


$$
10^{\ell} / 9 \leq a \cdot 10^{\ell} / 9=\left|\sigma\left(a \cdot 10^{\ell} / 9\right)\right|=\left|\sigma\left(g h(\alpha \beta)^{n-1}\right)\right|=\left|g_{2} h_{2}\left(\alpha_{2} \beta_{2}\right)^{n-1}\right|<4,
$$

where $g_{2}=g\left(\alpha_{2}, k\right)$ and $h_{2}=g\left(\beta_{2}, k+m\right)$ (both are smaller than 2). The previous inequality implies $10^{\ell} \leq 36$ and so $\ell=1$ yielding ta contradiction. Therefore, we have that $\Lambda \neq 0$ as desired.

In order to use Lemma 1 , we take $t:=3$,

$$
\gamma_{1}:=10, \gamma_{2}:=\alpha \beta, \gamma_{3}:=\theta_{a, m}
$$

and

$$
b_{1}:=\ell, b_{2}:=-n+1, b_{3}:=1 .
$$

For this choice, we have $D=[\mathbb{Q}(\alpha, \beta): \mathbb{Q}] \leq k(k+m) \leq k(k+9) \leq 6 k^{2}$, for $k \geq 2$. Note that $\mathrm{h}\left(\gamma_{1}\right)=\log 10$, and by Lemma 2

$$
\mathrm{h}\left(\gamma_{2}\right) \leq \mathrm{h}(\alpha)+\mathrm{h}(\beta)=\frac{\log \alpha}{k}+\frac{\log \beta}{k+m} \leq \frac{2 \log 2}{k} .
$$

Moreover, in [39] (p. 73), an estimate for $\mathrm{h}(g)$ was given. More precisely, it was proved that $\mathrm{h}(g)<\log (4 k+4)$. Thus, by using again Lemma 2, we have

$$
\mathrm{h}\left(\gamma_{3}\right) \leq \mathrm{h}(9 a)+\mathrm{h}(g)+\mathrm{h}(h) \leq \log (81)+\log (4 k+4)+\log (4 k+4 m+4)<11.3 \log k,
$$

where we used that $4 k+4 m+4 \leq 4 k+40 \leq k^{5.6}$, for all $k \geq 2$.

Thus, we can choose

$$
A_{1}:=6 k^{2} \log 10, A_{2}:=12 k \log 2, A_{3}:=67.9 k^{2} \log k .
$$

Note that $\max \left\{\left|b_{1}\right|,\left|b_{2}\right|,\left|b_{3}\right|\right\}=\max \{\ell, n-1\}$. However, by (3), we deduce that

$$
2^{2 n-4} \geq F_{n}^{(k)} F_{n}^{(k+m)}=a\left(\frac{10^{\ell}-1}{9}\right)>10^{\ell-1}
$$

yielding $n>1.6 \ell+0.3>\ell$ and so we can take $B=n$.

Now, we are in a position to use Lemma 1 which provides us (after some manipulations)

$$
\left|1-a(9 g h)^{-1}(\alpha \beta)^{n-1} 10^{\ell}\right|>\exp \left(-1.4 \cdot 10^{15} k^{9}(\log k)^{2} \log n\right) .
$$

By combining (10) and (11), we obtain

$$
\frac{n}{\log n}<4.7 \cdot 10^{16} k^{9}(\log k)^{2} .
$$

Since, for $x>e$, the function $x \mapsto x / \log x$ is increasing, then it is a simple exercise to show that

$$
\frac{x}{\log x}<A \text { implies that } x<2 A \log A \text {. }
$$

Thus, by using (13) in (12) for $x:=n$ and $A:=2.7 \cdot 10^{15} k^{9}(\log k)^{2}$, we get

$$
n<2\left(4.7 \cdot 10^{16} k^{9}(\log k)^{2}\right) \log \left(4.7 \cdot 10^{16} k^{9}(\log k)^{2}\right)<2.4 \cdot 10^{18} k^{9}(\log k)^{3},
$$

where

$$
\log (4.7)+16 \log 10+9 \log k+2 \log \log k<46 \log k
$$


for all $k \geq 2$.

In conclusion, we arrive at

$$
\ell<n<4.4 \cdot 10^{18} k^{9}(\log k)^{3}
$$

Now, the proof splits into two cases, as follows.

\subsection{The Case $k \leq 322$}

By using (14), we have that, if $k \leq 322$, then

$$
\ell<n<2.3 \cdot 10^{43} .
$$

Now, we desire to apply the reduction method (based on Lemma 3) to make the bounds much smaller. The further arguments work for $\Lambda>0$ and $\Lambda<0$ in a similar way. So, to avoid unnecessary repetitions, we shall consider only the case when $\Lambda>0$ (and then $\left.\Lambda<e^{\Lambda}-1\right)$. Thus, by (8), we have

$$
0<\Lambda=\ell \log 10-(n-1) \log (\alpha \beta)+\log \theta_{a, m}<96 \cdot \alpha^{-n} .
$$

We divide by $\log (\alpha \beta)$ to get

$$
0<\ell \gamma_{k, m}-(n-1)+\mu_{k, a, m}<32 \cdot(1.4)^{-n}
$$

where $\gamma_{k, m}:=\log 10 / \log (\alpha \beta)$ and $\mu_{k, a, m}:=\log \theta_{a} / \log (\alpha \beta)$.

We claim that $\gamma_{k, m}$ is irrational for any integer $k \geq 2$ (In fact, it suffices to notice that $(\alpha \beta)^{r}$ is irrational for all integer $r \geq 1$ ). Let $q_{v, k, m}$ be the denominator of the $v$-th convergent of the continued fraction of $\gamma_{k . m}$. After taking $M:=2.3 \cdot 10^{43}$, we use Mathematica to obtain

$$
\min _{(k, m) \in[2,322] \times[1,9]} q_{600, k}>6 M .
$$

Moreover

$$
q^{\prime}:=\max _{(k, m) \in[2,322] \times[1,9]} q_{600, k}<3.4 \cdot 10^{423} .
$$

Define $\epsilon_{k, a, m}:=\left\|\mu_{k, a, m} q_{600, k, m}\right\|-M\left\|\gamma_{k, m} q_{600, k, m}\right\|$, for $k \in[3,322]$ and $a, m \in[1,9]$. Then,

$$
\epsilon^{\prime}:=\min _{(k, a, m) \in[3,322] \times[1,9]^{2}} \epsilon_{k, a}>2.6 \cdot 10^{-8} .
$$

Observe that the conditions of Lemma 3 are fulfilled for $A=32$ and $B=1.4$ and hence there is no solution to inequality (15) (and then no solution to the Diophantine Equation (3)) for $n$ and $l$ satisfying

$$
\ell<M \text { and } n \geq \frac{\log \left(A q^{\prime} / \epsilon^{\prime}\right)}{\log B} .
$$

Since $\ell<n<M$, then

$$
n \geq \frac{\log \left(A q^{\prime} / \epsilon^{\prime}\right)}{\log B}<3155 .
$$

Therefore, the possible solutions of (3) are in the range $\ell, n \in[2,3155]$ (since $\ell>1$ was already studied) and $k \in[3,322]$. By using Mathematica, we can explicit $F_{n}^{(k)} F_{n}^{(k+m)}$ $\left(\bmod 10^{10}\right)$, for $n \in[3,3155], k \in[3,322]$ and $m \in[1,9]$. Now, we search for 10 digits repdigits among these remainders $\left(\bmod 10^{10}\right)$ and none a solution was found. This completes the proof. 


\subsection{The Case $k \geq 323$}

3.3.1. Bound for $k$ in Terms of $n$

In this case, we have

$$
n<4.4 \cdot 10^{18} k^{9}(\log k)^{3}<2^{k / 2}<2^{(k+9) / 2} .
$$

This allows us to apply the Bravo and Luca method (see [39]). The main idea of their approach is to approximate $\alpha$ and $\beta$ to 2 (in an explicit form). We shall omit the details since it is very well explained in [39]. Therefore, we can write

$$
g \alpha^{n-1}=2^{n-2}+\delta_{1} / 2+2^{n-1} \eta_{1}+\eta_{1} \delta_{1}
$$

and

$$
h \beta^{n-1}=2^{n-2}+\delta_{2} / 2+2^{n-1} \eta_{2}+\eta_{2} \delta_{2}
$$

where $\eta_{1}:=g-1 / 2, \eta_{2}:=h-1 / 2, \delta_{1}:=2^{n-1}-\alpha$ and $\delta_{2}:=2^{n-1}-\beta$. Moreover, they proved that

$$
\left|\eta_{i}\right|<2(k+i-1) / 2^{k+i-1} \text { and }\left|\delta_{i}\right|<2^{n+2} / 2^{(k+i-1) / 2} \text {, for } i \in\{1,2\} .
$$

Now, we can use all these information to derive (after many straightforward manipulations) that

$$
\begin{aligned}
& 2^{2 n-4}=\left(g \alpha^{n-1}-\delta_{1} / 2-2^{n-1} \eta_{1}-\eta_{1} \delta_{1}\right)\left(h \beta^{n-1}-\delta_{2} / 2-2^{n-1} \eta_{2}-\eta_{2} \delta_{2}\right)=g h(\alpha \beta)^{n-1}+t_{n, k \prime} \\
& \text { where }\left|t_{n, k}\right|<15 \cdot 2^{2 n-1} / 2^{k / 2}, \text { for } k \geq 323 \text {. Thus } \\
& \qquad\left|2^{2 n-4}-a \cdot \frac{10^{\ell}}{9}\right| \leq\left|g h(\alpha \beta)^{n-1}-a \frac{10^{\ell}}{9}\right|+\left|t_{n, k}\right|<3 \beta^{n-1}+15 \cdot \frac{2^{2 n-1}}{2^{k / 2}}<18 \cdot \frac{2^{2 n-1}}{2^{k / 2}},
\end{aligned}
$$

where we used the inequality (7). We divide the previous inequality by $2^{2 n-4}$ to have

$$
\left|1-\frac{16 a}{9} 10^{\ell} \cdot 4^{-n}\right|<\frac{144}{2^{k / 2}} .
$$

Clearly, the left-hand side above is nonzero (otherwise 5 would divide $9 \cdot 4^{n}$ ). We shall apply Lemma 1 again for $t:=3$,

$$
\gamma_{1}:=10, \gamma_{2}:=4, \gamma_{3}:=16 a / 9
$$

and

$$
b_{1}:=\ell, b_{2}:=-n, b_{3}:=1 .
$$

Therefore, $D=1$ and $\mathrm{h}\left(\gamma_{i}\right)=\log \gamma_{i}(i \in\{1,2\})$, and by Lemma $2, \mathrm{~h}\left(\gamma_{3}\right)<7.2$. Thus, we can choose

$$
A_{1}:=\log 10, A_{2}:=\log 4, A_{3}:=7.2
$$

and $B=n$ and Lemma 1 gives

$$
\left|1-\frac{16 a}{9} 10^{\ell} \cdot 4^{-n}\right|>\exp \left(-6.6 \cdot 10^{12} \log n\right) .
$$

By combining (16) and (17), we get

$$
k<1.8 \cdot 10^{13} \log n .
$$


3.3.2. Explicit Bounds for $n, \ell$ and $k$ and the Reduction Method

By (14) and (18), we have

$$
k<1.8 \cdot 10^{13} \log \left(4.4 \cdot 10^{18} k^{9}(\log k)^{3}\right) .
$$

Since the left-hand side is $O(k)$, while the right-hand one is $O(\log k)$, then the previous inequality is valid only for finitely many values of $k$ (since $k / \log k \rightarrow 0$ as $k \rightarrow \infty$ ). In order to make this explicitly, after some calculations, the previous inequality becomes

$$
k<5.2 \cdot 10^{19} \log k .
$$

Now, by using Mathematica software, we infer that $k<2.7 \cdot 10^{21}$ and $n<5.4 \cdot 10^{214}$. Therefore, we need to make these bounds smaller. For that, we shall use Lemma 3. Take $\Gamma:=(\ell-1) \log 10-n \log 4+\log (160 a / 9)$. By $(16)$,

$$
\left|e^{\Gamma}-1\right|<\frac{144}{2^{k / 2}}
$$

Again, we can suppose that $\Gamma>0$ (otherwise, use that $1-e^{-x}=\left|e^{x}-1\right|$, if $x<0$ ) to write

$$
0<(\ell-1) \log 10-n \log 4+\log (160 a / 9)<144 \cdot 2^{k / 2} .
$$

Dividing through by $\log 4$, we obtain

$$
0<(\ell-1) \gamma-n+\mu_{a}<104 \cdot(1.4)^{-k},
$$

where $\gamma:=\log 10 / \log 4$ and $\mu_{a}:=\log (160 a / 9) / \log 4$, for $a \in[1,9]$.

Clearly, $\gamma$ is irrational and Let $q_{v}$ be the denominator of the $v$-th convergent of its continued fraction. Taking $M:=3.4 \cdot 10^{214}$, we use Mathematica again to obtain that $q_{454}>6 \mathrm{M}$.

Define $\epsilon_{a}:=\left\|\mu_{a} q_{454}\right\|-M\left\|\gamma q_{454}\right\|$, for $a \in[1,9]$. Then, we obtain

$$
\epsilon^{\prime}:=\min _{a \in[1,9]} \epsilon_{a}>0.009
$$

Thus, all conditions of Lemma 3 are satisfied for $A=104$ and $B=1.4$ and hence there is no solution to inequality (19) for $\ell$ and $k$ satisfying

$$
\ell-1<M \text { and } k \geq \frac{\log \left(A q_{454} / \epsilon^{\prime}\right)}{\log B} .
$$

Since $\ell-1<n<M$, we have that $k<\frac{\log \left(A q_{454} / \epsilon^{\prime}\right)}{\log B}<1506$. Thus $n<3.8 \cdot 10^{49}$. By repeating this process again for the new $M=3.8 \cdot 10^{49}$ (we use $q_{120}$ ), we obtain $k \leq 365$ and $n<5.7 \cdot 10^{493}$. To conclude, we apply one more time Lemma 3 for the new choice of $M:=3.8 \cdot 10^{49}$ (for $q_{107}$ ) and hence $k \leq 322$.

This contradicts our assumption that $k \geq 323$. In conclusion, there is no solution to the Diophantine Equation (3) for $k \geq 323$.

\section{Other Similar Equations: The Elementary Method}

It is important to notice that an elementary method does not provide a reasonable approach to deal with the Equation (1). The possible reasons can be because the product (in the left-hand side) possesses only two terms, $n$ can be much larger than $k+m$ as well as the very limited knowledge about arithmetic properties of repdigits (from that equation we infer only that $\ell$ must be a composite number).

However, we shall provide here some similar Diophantine equations which can be solved by using basic tools.

The first one is when the order is larger than the index. More precisely 
Proposition 1. The Diophantine equation

$$
F_{n}^{(k+n)}=a\left(\frac{10^{\ell}-1}{9}\right)
$$

does not have a solution in positive integers $n, a, k, \ell$, with $k \geq 2, \ell>1$ and $a \in[1,9]$.

The proof follows because $F_{n}^{(k+n)}=2^{n-2}$, for all $n \in[2, k+n+1]$ and so $F_{n}^{(k+n)} \equiv 0$ $(\bmod 16)$, for all $n \geq 6$. Since none repdigit is a multiple of 16 , then $n \in[1,5]$. However, $F_{5}^{(k+5)}<10$ which contradicts $\ell>1$.

Another possible problem is a symmetric equation (between $k$ and $n$ ) which forces the previous case. For example:

Proposition 2. The Diophantine equation

$$
F_{n}^{(k)} F_{k}^{(n)}=a\left(\frac{10^{\ell}-1}{9}\right),
$$

does not have a solution in positive integers $n, a, k, \ell$, with $k \geq 2, \ell>1$ and $a \in[1,9]$.

The proof is similar to the previous one by using that either $n \leq k+1$ or $k \leq n+1$.

In the previous propositions, we used the fact that $F_{n}^{(k)}$ is a power of 2 , when $n \leq k+1$. However, we can use another approach (since $F_{n}^{(k)}$ is never a power of two, for $n>k+1$, see [39]) for equations related to the product of "many" consecutive $k$-bonacci numbers. More precisely:

Proposition 3. The Diophantine equation

$$
F_{n}^{(k)} F_{n+1}^{(k)} \cdots F_{n+4 k}^{(k)}=a\left(\frac{10^{\ell}-1}{9}\right)
$$

does not have a solution in positive integers $n, a, k, \ell$, with $k \geq 2, \ell>1$ and $a \in[1,9]$.

For proving this, we notice that the recurrence of $\left(F_{n}^{(k)}\right)_{n}$ yields

$$
F_{n+1}^{(k)}=2 F_{n}^{(k)}-F_{n-k}^{(k)} .
$$

Thus, $F_{n+(k+1)}^{(k)} \equiv F_{n}^{(k)}(\bmod 2)$, i.e., $\left(F_{n}^{(k)}\right)_{n}$ is a $(k+1)$-periodic sequence $(\bmod 2)$. Thus, the sequence $\left(F_{n}^{(k)}\right)_{n}$ contains infinitely many even numbers (for example, $F_{3+t(k+1)^{\prime}}^{(k)}$ for all $t \geq 0$ ). For this reason, at least one among the numbers $F_{n}^{(k)}, F_{n+1}^{(k)}, \ldots, F_{n+k}^{(k)}$ is even. The same happens for the lists $\left\{F_{n+k+1}^{(k)}, \ldots, F_{n+2 k}^{(k)}\right\},\left\{F_{n+2 k+1}^{(k)}, \ldots, F_{n+3 k}^{(k)}\right\}$ and $\left\{F_{n+3 k+1}^{(k)}, \ldots, F_{n+4 k}^{(k)}\right\}$. Hence, the product

$$
F_{n}^{(k)} F_{n+1}^{(k)} \cdots F_{n+4 k}^{(k)}
$$

is a multiple of 16 and so it can not be a repdigit.

\section{Conclusions}

For any integer $k \geq 2$, the sequence of the $k$-generalized Fibonacci numbers (or $k$ bonacci numbers) is defined by the $k$ initial values $F_{-(k-2)}^{(k)}=\cdots=F_{0}^{(k)}=0$ and $F_{1}^{(k)}=1$ and such that each term afterwards is the sum of the $k$ preceding ones. In this paper, we search for solutions of the Diophantine equation $F_{n}^{(k)} F_{n}^{(k+m)}=a\left(10^{\ell}-1\right) / 9$ for positive 
integers $k, n, \ell, m$ and $a$, with $k \geq 2, \ell \geq 2$ and $a, m \in[1,9]$. In particular, the only repdigits, which can be written as a product of $n$th terms of two generalized Fibonacci sequences with consecutive orders, has only one digit. Our approach to proving this fact is to combine the Baker's theory (on lower bounds for linear forms in the logarithms) with a reduction method from the theory of continued fractions (due to Dujella and Pethó). In the concluding section, we present some similar problems which can be solved by using only elementary tools.

Author Contributions: P.T. conceived the presented idea on the conceptualization, methodology, and investigation. Writing-review and editing and preparation of program procedures in Mathematica were done by P.C. All authors have read and agreed to the published version of the manuscript.

Funding: The authors was supported by the Project of Specific Research PrF UHK no. 2101/2021, University of Hradec Králové, Czech Republic.

Institutional Review Board Statement: Not applicable.

Informed Consent Statement: Informed consent was obtained from all subjects involved in the study.

Conflicts of Interest: The authors declare no conflict of interest.

\section{References}

1. Koshy, T. Fibonacci and Lucas Numbers with Applications; Wiley: New York, NY, USA, 2001.

2. Vorobiev, N.N. Fibonacci Numbers; Birkhäuser Verlag: Basel, Switzerland, 2002.

3. Kalman, D.; Mena, R. The Fibonacci Numbers-Exposed. Math. Mag. 2003, 76, 167-181.

4. Ballew, D.W.; Weger, R.C. Repdigit Triangular Numbers. J. Recreat. Math. 1975, 8, 96.

5. Keith, M. On Repdigit Polygonal Numbers. J. Integer Seq. 1998, 1, 6.

6. Díaz Alvarado, S.; Luca, F. Fibonacci numbers which are sums of two repdigits. In Proceedings of the XIVth International Conference on Fibonacci Numbers and Their Applications, Morelia, Mexico, 1-7 July 2011; pp. 97-111.

7. Luca, F. Repdigits as sums of three Fibonacci numbers. Math. Commun. 2012, 17, 1-11.

8. Faye, B.; Luca, F. Pell and Pell-Lucas numbers with only one distinct digit. Ann. Math. Inform. 2015, 45, 55-60.

9. Marques, D. On k-generalized Fibonacci numbers with only one distinct digit. Util. Math. 2015, 98, 23-31.

10. Bravo, J.J.; Luca, F. Repdigits as sums of two k-Fibonacci numbers. Monatshefte Math. 2015, 176, 31-51. [CrossRef]

11. Bravo. J.J.; Faye, B.; Luca, F.; Tall, A. Repdigits as Euler functions of Lucas numbers. An. Univ. Ovidius Constanta 2016, 24, 105-126.

12. Normenyo, B.V.; Luca, F.; Togbé, A. Repdigits as sums of three Pell numbers. Period. Math. Hung. 2018, 77, 318-328. [CrossRef]

13. Luca, F.; Normenyo, B.V.; Togbé, A. Repdigits as sums of four Pell numbers. Bol. Soc. Mat. Mex. 2019, 25, 249-266. [CrossRef]

14. Luca, F.; Normenyo, B.V.; Togbé, A. Repdigits as sums of three Lucas numbers. Colloq. Math. 2019, 156, 255-265. [CrossRef]

15. Sahukar, M.K.; Panda, G.K. Repdigits in Euler functions of Pell numbers. Fibonacci Quart. 2019, 57, $134-138$.

16. Lomelí, A.C.G.; Hernandé, H.S. Repdigits as Sums of Two Padovan Numbers. J. Integer Seq. 2019, $22,3$.

17. Şiar, Z.; Erduvan, F.; Keskin, R. Repdigits as product of two Pell or Pell-Lucas numbers. Acta Math. Univ. Comen. 2019, 88, 247-256.

18. Bitim, B.D. On the Diophantine equation $L_{n}-L_{m}=2 \cdot 3^{a}$. Period. Math. Hung. 2019, 79, $210-217$.

19. Trojovský, P. On Diophantine equations involving Lucas sequences. Open Math. 2019, 17, 942-946. [CrossRef]

20. Trojovský, P. On Terms of Generalized Fibonacci Sequences which are Powers of their Indexes. Mathematics 2019, 7, 700. [CrossRef]

21. Alahmadi, A.; Altassan, A.; Luca, F.; Shoaib, H. Fibonacci numbers which are concatenations of two repdigits. Quaest. Math. 2019. [CrossRef]

22. Keskin, R.; Erduvan, F. Repdigits in the base $b$ as sums of four Balancing numbers. Math. Bohem. 2020. [CrossRef]

23. Panda, G.K.; Sahukar, M.K. Repdigits in Euler functions of associated Pell numbers. Proc. Math. Sci. 2020, 130, 25. [CrossRef]

24. Adegbindin, C.; Luca, F.; Togbé, A. Pell and Pell-Lucas numbers as sums of two repdigits. Bull. Malays. Math. Sci. Soc. 2020, 43, 1253-1271. [CrossRef]

25. Trojovský, P. Fibonacci numbers with a prescribed block of digits. Mathematics 2020, 8, 639. [CrossRef]

26. Ddamulira, M. Repdigits as sums of three Padovan number. Bol. Soc. Mat. Mex. 2020, 26, 1-15. [CrossRef] [PubMed]

27. Erduvan, F.; Keskin, R.; Şiar, Z. Repdigits base $b$ as products of two Lucas numbers. Quaest. Math. 2020. [CrossRef]

28. Erduvan, F.; Keskin, R. Repdigits as products of two Fibonacci or Lucas numbers. Proc. Indian Acad. Sci. Math. Sci. 2020, 130, 28. [CrossRef]

29. Qu, Y.; Zeng, J. Lucas Numbers Which Are Concatenations of Two Repdigits. Mathematics 2020, 8, 1360. [CrossRef]

30. Ddamulira, M. Repdigits as sums of three Balancing numbers. Math. Slovaca 2020, 70, 557-566 [CrossRef]

31. Ddamulira, M. Tribonacci numbers that are concatenations of two repdigits. Rev. Real Acad. Cienc. Exactas Fis. Nat. Ser. A Mat. 2020, 114. [CrossRef] 
32. Rayaguru, S.G.; Panda, G.K. Balancing numbers which are concatenation of two repdigits. Bol. Soc. Mat. Mex. 2020, 26, 911-919. [CrossRef]

33. Erduvan, F.; Keskin, R. Lucas numbers which are concatenations of three repdigits. Results Math. 2021, 76, 13. [CrossRef]

34. Luca, F. Fibonacci and Lucas numbers with only one distinct digit. Port. Math. 2000, 57, 243-254.

35. Marques, D. On the intersection of two distinct $k$-generalized Fibonacci sequences. Math. Bohem. 2012, 137, 403-413. [CrossRef]

36. Bednařík, D.; Trojovská, E. Repdigits as Product of Fibonacci and Tribonacci Numbers. Mathematics 2020, 8, 1720. [CrossRef]

37. Trojovský, P. On Repdigits as Sums of Fibonacci and Tribonacci Numbers. Symmetry 2020, 12, 1774. [CrossRef]

38. Dresden, G.P.; Du, Z. A simplified Binet formula for k-generalized Fibonacci numbers. J. Integer Seq. 2014, 17, 3.

39. Bravo, J.J.; Luca, F. Powers of two in generalized Fibonacci sequences. Rev. Colomb. Mat. 2012, 46, 67-79.

40. Matveev, E.M. An explicit lower bound for a homogeneous rational linear form in logarithms of algebraic numbers II. Izv. Math. 2000, 64, 1217-1269. [CrossRef]

41. Bugeaud, Y.; Mignotte, M.; Siksek, S. Classical and modular approaches to exponential Diophantine equations. I. Fibonacci and Lucas perfect powers. Ann. Math. 2006, 163, 969-1018. [CrossRef]

42. Waldschmidt, M. Diophantine Approximation on Linear Algebraic Groups; Springer: Berlin/Heidelberg, Germany; New York, NY, USA, 2000.

43. Dujella, A.; Pethő, A. A generalization of a theorem of Baker and Davenport. Q. J. Math. Oxf. Ser. 1998, 49, 291-306. [CrossRef]

44. Bravo, J.J.; Luca, F. On a conjecture about repdigits in k-generalized Fibonacci sequences. Publ. Math. Debr. 2013, 82, 623-639. [CrossRef] 